\title{
Complex trajectory method in semiclassical propagation of wave packets
}

\author{
Marc Boiron and Maurice Lombardi \\ Laboratoire de Spectrométrie Physique, Université Joseph-Fourier-Grenoble, BP87, \\ F-38402 Saint-Martin-d'Hères Cédex, France
}

(Received 19 March 1997; accepted 24 November 1997)

\begin{abstract}
We propose a semiclassical wave packet propagation method relying on classical trajectories in a complex phase space. It is based on the Schrödinger wave equation and the usual expansion with respect to $\hbar$, except that the amplitude of the wave packet is taken into account at the very zeroth order, unlike in the usual WKB method where it is treated as a corrective or first order term. Formally, it amounts to making both the wavelength and the width of the wave packet tend to zero with $\hbar$. The action and consequently the classical trajectories derived are complex. This method is tested successfully in many cases, analytically or numerically, including the bounce and even the splitting of the wave packet. Our method appears to be much more accurate than the WKB method while less computationally demanding than the Van-Vleck formula. Moreover, it has a particularly interesting property: the singularities (caustics) of the usual semiclassical theories do not appear in this formalism in all cases tested. (C) 1998 American Institute of Physics.
\end{abstract}

[S0021-9606(98)00309-2]

\section{INTRODUCTION: THE SEMICLASSICAL APPROXIMATION IN TIME-DEPENDENT PROBLEMS}

Quantum cross-section calculations in collisions involving atoms or molecules can be carried out in two different frameworks: a time-independent framework or timedependent framework. The early means of calculation was to use the time-independent scheme, that is the so-called "close-coupling'" method. Its numerical cost is high, proportional to $N^{3}$ where $N$ is the number of rovibrational channels included in the simulated model. Current computer power limits this number to a few thousand. To go further, timedependent or wave packet propagation methods were later adopted. Their numerical cost is less since they allow calculations to be made on a restricted portion of the $N$ channels at a time. These methods rely on some discretization of the initial partial differential equation of propagation. The cost is then directly related to the size of the grid required. This size (number of points) depends on many factors that subsequently are limitations to numerical simulations.

(1) The number of electronic levels. In this paper we will only deal with elastic processes so one surface of potential is assumed.

(2) The number $d$ of degrees of freedom: The grid size is a function of the power of $d$.

(3) The average kinetic energy $K$. Since the order of magnitude of the wavelength is given by the de Broglie relation $\lambda_{D B}=\hbar / p$, the steps of the grid, of order $\lambda_{D B}$, shall thus be smaller the larger the average momentum. Consequently, the grid size increases as $\sqrt{K}$.

A compromise between these factors should be made in order to fit the numerical task to computer possibilities. Current achievements of wave packet propagation show that the number of degrees of freedom is limited to 3 if the process involves a "high" (few eV) vibrational energy such as in
$\mathrm{O}_{3}{ }^{1}$ or the $\mathrm{H}+\mathrm{O}_{2}$ reaction; ${ }^{2}$ or 6 (4 atoms) if the simulation is restricted to the lowest lying states, that is $\nu=0,1$ vibrational quantum initially. 3,4

Besides these "brute-force" methods, approximate methods were developed, mainly in order to outstrip previously mentioned bounds and to give a simplified image of the process by avoiding the exclusive use of quantum mechanics theory. Most of these approximate methods rely entirely or partially on classical mechanics. The reasons for this are obvious: Classical mechanics provides a handy view of processes and technically the equations of classical mechanics (canonical equations of Hamilton) are ordinary differential equations, not partial differential equations such as the Schrödinger equation; they are thus far more easy to integrate numerically.

We can distinguish three stages of approximation:

(1) Purely classical. The original quantal problem is replaced by a classical problem: The motion of the nuclei belongs to classical trajectories. ${ }^{5}$ Possible nonadiabatic transitions are taken into account by the Landau-Zener formula. $^{6}$

(2) Hemiquantal. The degrees of freedom are divided into two sets: one treated classically as previously, and the other quantally. 7,8

(3) Semiclassical. The physical concepts used are those of quantum mechanics: wave functions or propagator, but they are calculated by means of classical trajectories. ${ }^{9}$ The most simple example of this is the WKB approximation of a one-dimensional time independent wave function. $^{10}$

Let us now describe the existing semiclassical theories, and discuss their advantages and drawbacks.

The first of them starts with the differential point of view of quantum mechanics (Schrödinger equation) and the rewriting of the wave function as an envelope times a purely 
oscillating function: ${ }^{11} A e^{i S}$. At the semiclassical limit, the envelope $A$, the local de Broglie wavelength $\lambda=h /\|\nabla S\|$ and the potential vary slowly on the scale of the de Broglie wavelength itself. This allows one the derivation of two decoupled equations: The first is the classical Hamilton-Jacobi equation that is satisfied by $S$, the second is a continuity equation satisfied by $A$. Solving these equations is much less demanding than solving the original Schrödinger equation since $S$ $=\int L d t$ and $A=A(0) \exp \left(-\int \nabla^{2} S / 2 m d t\right)$ along classical trajectories ( $L$ is the classical Lagrangian). Moreover, only one trajectory calculation is required to compute the propagated wave function at one point. However, the accuracy of this approximation is very poor: In the most simple case of a Gaussian wave packet moving in a flat potential, it is easy to show that this method gives a constant width wave packet whereas the quantum wave packet spreads with time. Considering general potentials, the semiclassical limit obtained by such a procedure entails singularities (caustic curves) which cause the divergence of $\nabla^{2} S$, stopping the integration of $A$ at a finite time. An extension of this method ${ }^{12}$ is obtained through the $\hbar$-expansion of the functions $S$ or $A$, giving the same result as previously up to first order. These formalisms will be referred to as the WKB method.

The second widely used semiclassical theory relies on the Feynman's formulation of quantum mechanics, based on path integrals for the propagator. By retaining virtual trajectories nearby to classical ones, it is possible to derive an approximate form for the propagator, known as the VanVleck-Gutzwiller formula. ${ }^{13}$ While this method is more accurate than the previous one - it is exact for polynomial potentials up to second degree - it can hardly be used to propagate wave packets numerically because of the integrations required. Many classical trajectories have to be determined by a root searching procedure in order to compute the amplitude of the propagated wave packet at one point. These calculations shall be repeated for every point where the amplitude is needed. Within this framework difficulties due to caustics arise too, they can be partially overcome by the use of Morse's indices.

The subject of wave packet propagation at the semiclassical limit has been extensively studied by E. J. Heller and co-workers since 1975, whose early method is the wellknown Gaussian wave packet propagation. ${ }^{14}$ Heller showed ${ }^{15}$ that it is a consequence of the Van-Vleck formula in the case where the action can be approximated as a second order expansion with respect to the end points of the classical trajectories needed to compute the propagated wave packet. Assuming the initial wave packet to be Gaussian, it is then possible to compute analytically the propagated wave packet that remains Gaussian with time, getting rid of singularities. Improvements of this basic method were made, ${ }^{16}$ which are called nonlinear methods. ${ }^{17}$

As a conclusion, we have on one hand the simple but inaccurate semiclassical WKB method, and on the other hand, the Van-Vleck formula, more accurate but computationally more demanding. The approximations, i.e., the semiclassical limits both these methods provide, entail singularities (caustics).

In this paper, we propose and test a method devoted to the propagation of wave packets at the semiclassical limit. It is based on a WKB-like procedure of an expansion with respect to a small parameter. We will focus here on the Schrödinger equation, for which $\hbar$ is the right ordering parameter in the expansion, even if the method is general. The essential difference with the usual WKB method is that the procedure we propose treats on equal footing phase and amplitude of the wave function, that is, at the very zeroth order, whereas the usual WKB method deals with the amplitude as a first order or corrective term to the zeroth order approximation which concerns the phase only. From a formal point of view, we will show that it amounts to making not only the wavelength tend to zero with the expansion parameter but the spatial extension of the wave packet as well, unlike the WKB method. A prominent consequence of this is the necessity to take into account classical trajectories in a phase space made of complex numbers, hence the name of "complex trajectory method." In return, while remaining in the simple WKB-like framework of the semiclassical approximation of the wave function itself, with the advantages quoted before, a large increase in accuracy of the semiclassical approximation is obtained compared to the usual WKB method. An unexpected and particularly appealing property of our formalism is observed: In the examples detailed later, no caustics were encountered whereas all other real semiclassical theories give rise to such singularities.

This paper is divided as follows: Section II is devoted to an explanation of the general procedure leading to the computation of the semiclassical limit by means of complex trajectories. The following sections are devoted to comparison in many potentials between exact propagated quantum wave packets and semiclassical wave packets obtained by the method stated previously. Section III is devoted to the study of analytically soluble potentials, where the trajectories, hence the semiclassical wave packets, can be formally exactly calculated as well as the quantum wave packet. In Section IV numerical trajectory calculations are carried out as well as numerical resolutions of the Schrödinger equation.

\section{PRINCIPLE}

\section{A. General procedure}

The method we propose can be applied to any single linear partial differential equation (PDE) in a multidimensional space. Since the illustrating examples given below will concern quantum wave packet propagation only, we restrict the statement of the method to the time-dependent Schrödinger equation. Thus, we look for an approximate solution of the equation:

$$
i \hbar \frac{\partial}{\partial t} \psi(\mathbf{x}, t)=\hat{H} \psi(\mathbf{x}, t)=\left(-\frac{\hbar^{2}}{2 m} \nabla^{2}+V(\mathbf{x})\right) \psi(\mathbf{x}, t) .
$$

where $\psi(\mathbf{x}, t)$ is the unknown wave function of the position variables $\mathbf{x}=\left(x_{1}, \ldots, x_{d}\right)-d$ is the number of degrees of freedom - and time $t$. The operator $\hat{H}$ is the quantum ana$\log$ of the classical Hamiltonian $H$ reading:

$$
H(\mathbf{x}, \mathbf{p})=\frac{\mathbf{p}^{2}}{2 m}+V(\mathbf{x}) \text {. }
$$


The initial condition of Eq. (1) is given at the initial time $t$ $=0$, that is,

$$
\psi(\mathbf{x}, 0)=\psi_{\text {init }}(\mathbf{x}),
$$

where $\psi_{\text {init }}$ is assumed to be a localized wave function. We pose for convenience:

$$
\psi_{\text {init }}(\mathbf{x})=\varrho(\mathbf{x}) e^{i \varphi(\mathbf{x})}=e^{i \Phi(\mathbf{x})},
$$

where the modulus $\varrho$ and the phase $\varphi$ are real values whereas $\Phi$ is a complex value.

Like in the usual WKB method, we write the wave function as an expansion with respect to $\hbar$ under the form of the "Ansatz":

$\psi(\mathbf{x}, t)=\exp \left(i\left(S_{0}(\mathbf{x}, t)+\hbar S_{1}(\mathbf{x}, t)+\hbar^{2} S_{2}(\mathbf{x}, t)+\cdots\right) / \hbar\right)$.

Combining this expression with the Schrödinger equation (1) and equating each coefficient of the resulting $\hbar$ expansion leads to equations satisfied by the $S_{j}$ functions. Before deriving them, we first look at the initial condition that these functions shall satisfy. They are obviously related to the initial condition of the original wave equation (3).

In the WKB method, following the physical idea that, at the semiclassical limit, the amplitude of the wave function is a slowly varying envelope with respect to its oscillations, one imposes the first order corrective term to be identified with the amplitude of the wave packet at the starting time, i.e.,

$$
\begin{aligned}
& S_{0}(\mathbf{x}, 0) / \hbar=\varphi(\mathbf{x}), \\
& \exp \left(i S_{1}(\mathbf{x}, 0)\right)=\varrho(\mathbf{x}), \\
& S_{j}(\mathbf{x}, 0)=0, \quad \forall j \geqslant 2 .
\end{aligned}
$$

In doing this, one shows then that $S_{0}$ satisfies the HamiltonJacobi equation of the classical Hamiltonian associated with the Schrödinger equation (1).

In our method, instead of the above, we do not make a distinction between phase and amplitude, consequently, we identify the whole initial wave function $\psi_{\text {init }}(\mathbf{x})$ with $e^{i S_{0} / \hbar}$ at the starting time, putting the other terms equal to zero, i.e.,

$$
\begin{aligned}
& S_{0}(\mathbf{x}, 0) / \hbar=\Phi(\mathbf{x}), \\
& S_{j}(\mathbf{x}, 0)=0, \quad \forall j \geqslant 1 .
\end{aligned}
$$

Our study focuses on wave packet propagation, as a consequence $\psi(\mathbf{x}, t)$ is a localized wave function of $\mathbf{x}$. It is then clear that the function $\Phi$ contains both a real and an imaginary part, at starting time and later. The real part is a phase term, typically of the linear form $\mathbf{k} \cdot \mathbf{x}$, giving the approximate spatial frequency $\mathbf{k} / 2 \pi$. The imaginary part provides the necessary limitation of the width of the wave packet in space. Propagating wave packets at the semiclassical limit, by means of the expansion (5) and the new conditions (9)(10) leads us thus to introduce a complex generalized action $S_{0}(\mathbf{x}, t)$. The originality of our method is thus clear at this stage: not only the phase but also the amplitude are treated at the zeroth order. The consequence of this on the formal way of taking the semiclassical limit that this method implies will be examined later, as well as its remarkable accuracy property. In particular, taking Eqs. (9) and (10) instead of Eqs.
(6)-(8) has much more profound consequences than a mere shift of quantities among the terms of the expansion.

We now carry out the expansion in $\hbar$ of the wave equation (1) with its wave function rewritten as Eq. (5).

\section{B. Zeroth order: complex trajectories}

The zeroth order in $\hbar$ gives the usual Hamilton-Jacobi equation (HJE) associated with the classical Hamiltonian $H$, as expected:

$$
\begin{aligned}
J\left(\mathbf{x}, \partial_{\mathbf{x}} S_{0}, t, \partial_{t} S_{0}\right) & =\frac{1}{2 m}\left(\partial_{\mathbf{x}} S_{0}\right)^{2}+V(\mathbf{x})+\partial_{t} S_{0} \\
& =H\left(\mathbf{x}, \partial_{\mathbf{x}} S_{0}\right)+\partial_{t} S_{0}=0 .
\end{aligned}
$$

At this order, the consequence of the semiclassical approximation is to replace the original second order PDE (1) by the Hamilton-Jacobi equation (11) that has the crucial property of being a single first order PDE. This is, in our sense, the essential property a semiclassical theory should lead with (when starting from the Schrödinger equation, not the propagator). Indeed, problems expressed in terms of PDEs can be recast under the form of a set of ordinary differential equations (ODEs) only in the particular case of a single first order PDE. In this case, it is possible to solve the Cauchy's problem by means of characteristic curves, see the clear reference of Arnold ${ }^{18}$ upon which this work is based. To be brief, let us say that these curves satisfy a particular set of ODEs, the characteristic equations (CEs) derived from the first order PDE, and they make a set covering the entire space of the variables of the unknown function. On each of these curves or 1D subspaces, the unknown function satisfies an ODE too. Then the original PDE can be exactly reformulated in terms of a set of ODEs. As ODEs are the equations of classical mechanics, we can say that the preceding transformation recasts the initial wave problem into a mechanical problem, possibly in a generalized sense. In the WKB theory, the characteristic curves exhibited are the classical trajectories of the corresponding classical Hamiltonian or the light rays when applied to optics. Note that the original wave equation is linear whereas the resulting HJE is nonlinear.

The peculiarity of our approach is that the "generalized" action $S_{0}$ is complex at the starting time. Because of the continuity of $S_{0}$ relatively to time, $S_{0}(\mathbf{x}, t)$ is a complexvalued function. Now the Hamilton-Jacobi equation (11) shall be considered as a complex equation. A natural starting point to solve it would be to separate the unknown function $S_{0}$ into its real and imaginary parts, in order to derive two real equations. This would lead to two coupled equations that would prevent one from using the theory of characteristics to solve them since this theory can be applied only to single equations. It is thus necessary to keep Equation (11) as a single complex scalar equation and extend formally the theory of characteristics to complex functions. As a consequence which we will make clear soon, this requires taking the derivative with respect to complex variables, that is dealing with a generalized configuration space extended to complex values. Then $S_{0}$ and $J$ shall be analytical functions of 
multiple complex variables. We assume in the following that these conditions are fulfilled. We will return to that question later.

The usual (real) theory of characteristics requires the propagation of trajectories in a space in which the natural space of the variables of the first order PDE (the HJE) is embedded: the complete phase space. It is made of the configuration space and time (i.e. the variables of the HJE), and the following associated variables $p_{k}$ and $\tau$, defined as

$$
\begin{aligned}
& p_{k}=\frac{\partial S_{0}}{\partial x_{k}}, \\
& \tau=p_{0}=\frac{\partial S_{0}}{\partial t} .
\end{aligned}
$$

These variables are the (generalized) momenta associated respectively with position and time. This set of variables, usually taken as real, is now considered as complex since $S_{0}$ is complex.

Solving the HJE (11) by means of the theory of characteristics ${ }^{18}$ enables us to write the following ODEs:

$$
\begin{aligned}
& \frac{d x_{k}}{d s}=\frac{\partial J}{\partial p_{k}}=\frac{\partial H}{\partial p_{k}}=\frac{p_{k}}{m}, \\
& \frac{d p_{k}}{d s}=-\frac{\partial J}{\partial x_{k}}=-\frac{\partial H}{\partial x_{k}}=-\frac{\partial V}{\partial x_{k}}, \\
& \frac{d t}{d s}=\frac{\partial J}{\partial \tau}=1, \\
& \frac{d \tau}{d s}=-\frac{\partial J}{\partial t}=0, \\
& \frac{d S_{0}}{d s}=\sum_{k} p_{k} \frac{\partial J}{\partial p_{k}}+\tau \frac{\partial J}{\partial \tau} .
\end{aligned}
$$

The first equations (first two columns) of each line result from the theory of characteristics, the following ones from the peculiar form of the function $J$ (11). The first four lines (14)-(17) are the equations of the characteristic curves in the complete phase space, whose parameter is $s$. Equation (18) gives the value of the unknown function $S_{0}$ along these curves. Note that although the momenta variables are taken as independent, the solutions given by the preceding equations satisfy the definition relations (12)-(13).

Since $t$ is the variable propagated in the original wave equation (1), it is better to substitute $s$ by $t$. This can be done provided that $d t / d s \neq 0$, whatever the value of $s$ and the characteristics considered, ${ }^{19}$ which is here trivially fulfilled, cf. Eq. (16). The previous equations read, in vectorial notation:

$$
\begin{aligned}
& \frac{d \mathbf{x}}{d t}=\frac{\mathbf{p}}{m}, \\
& \frac{d \mathbf{p}}{d t}=-\nabla V(\mathbf{x}), \\
& \frac{d S_{0}}{d t}=\frac{\mathbf{p}^{2}}{m}-E_{0},
\end{aligned}
$$

where $E_{0}=-\tau=$ constant along a trajectory because of Eq. (17). We recognize then the canonical equations of Hamilton associated with the classical Hamiltonian $H$ (2), hence the same notation $\mathbf{p}$ used for the conjugate variables of $\mathbf{x}$ and for $\partial_{\mathbf{x}} S_{0}$ (which are exactly equal to the momenta in the WKB theory).

Let us note that the natural set of variables of $S_{0}$ is $(\mathbf{x}, t)$. The method of characteristics, through ODEs (19)-(21), substitutes temporarily this set by $\left(\mathbf{x}_{0}, t\right)$, where $\mathbf{x}_{0}$ is the starting point of the curve at the initial time $t=0$. To illustrate; If the set of characteristics are viewed as a flow, the first variables are Lagrangian whereas the second are Eulerian. Further in the paper, no specific notation will be used, the variables being obvious by context.

We need the initial conditions of the CEs (19)-(21), they are naturally derived from the initial value of $S_{0}$ given by Eq. (9) and the relations (12)-(13). Let $\mathbf{x}_{0}$ be a starting position of a characteristics at time $t=0$, then the corresponding momenta are

$$
p_{k}\left(\mathbf{x}_{0}, 0\right)=\left(\frac{\partial S_{0}(\mathbf{x}, 0)}{\partial x_{k}}\right) .
$$

The HJE (11), taken at time $t=0$, allows the derivation of the initial value $\tau\left(\mathbf{x}_{0}, 0\right)$, as a function of $\mathbf{x}_{0}$ and $p_{k}\left(\mathbf{x}_{0}, 0\right)$, here it reads simply:

$$
-\tau\left(\mathbf{x}_{0}, 0\right)=E_{0}\left(\mathbf{x}_{0}\right)=H\left(\mathbf{x}_{0}, \mathbf{p}\left(\mathbf{x}_{0}, 0\right)\right) .
$$

Since $S_{0}\left(\mathbf{x}_{0}, 0\right)$ is complex, $p_{k}\left(\mathbf{x}_{0}, 0\right)$ and $E_{0}\left(\mathbf{x}_{0}\right)$ are necessarily complex, even for real values of $\mathbf{x}_{0}$. Let us stress the fact that the complex nature of these quantities results from the fact that the amplitude is included in the "action" $S_{0}$. It does not result, as in some variant of the WKB theory ${ }^{10}$, from the analytical extension of the action which remains real anyway for real values of the position.

From the initial conditions (22)-(23), it is obvious that the CEs (19)-(20) lead the position component $\mathbf{x}$ of the characteristics to be in the complex phase space since $p_{k}\left(\mathbf{x}_{0}, 0\right)$ is complex, even if the initial position $\mathbf{x}_{0}$ and the HamiltonJacobi function $J$ are real. Hence the name "complex trajectory method" attributed to this semiclassical theory. As a consequence, the equation of propagation (21) gives the value of $S_{0}$ for uninteresting complex $\mathbf{x}$ positions. The hypothesis of analyticity of the function $\mathbf{x} \mapsto S_{0}(\mathbf{x}, t), \forall t$ allows one to show that the propagation of the CEs gives the solution of the HJE. Two procedures will be described and discussed from a practical (numerical) point of view. For the sake of easy understanding, one can assume one is first dealing with the one-dimensional $(d=1)$ case.

(1) Let us suppose that $\mathbf{x}_{0}$ is a real vector belonging to the real space $\gamma$ of dimension $d$. The image of $\gamma$ through the propagation of the CEs (19)-(20) is a complex space $\gamma(t)$ of the same dimension $d$, see Fig. 1(a) in the case $d=1$. The property just mentioned allows one to extend analytically $\mathbf{x} \mapsto S_{0}(\mathbf{x}, t)$ from $\gamma(t)$ to the entire space $\mathbb{C}^{d}$, generalized position space, in order to recover $S_{0}(\mathbf{x}, t)$ with real $\mathbf{x}$ values. From a formal point of view, this means the following: once the system of CEs (19)-(21) is solved, the initial position $\mathbf{x}_{0}$ is eliminated between the equation of the trajectories $\mathbf{x}\left(\mathbf{x}_{0}, t\right)$ 

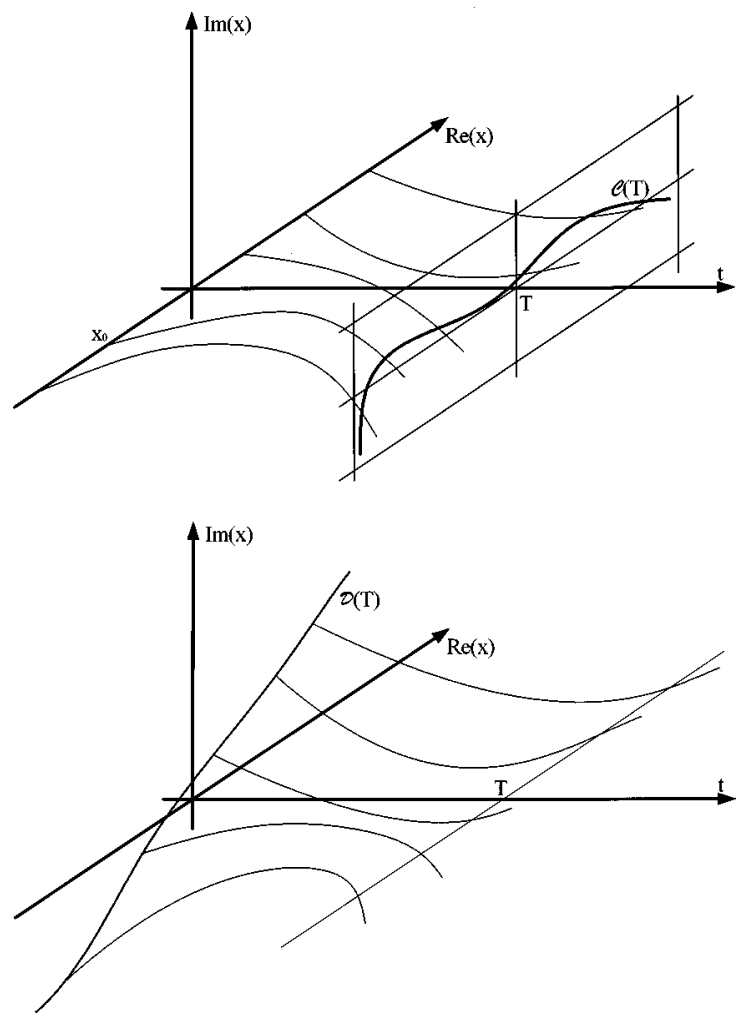

FIG. 1. (a) Some characteristic curves in the configuration/time space $(x$ $\in \mathrm{C}, t \in \mathrm{R})$ starting from a real coordinate point. The complex curve $\mathscr{C}(T)$ is the intersection of these trajectories with the plane $t=T$. Stated otherwise, $\mathscr{C}(T)$ is the image of the real line through the Hamiltonian flow. (b) The complex trajectories start from the curve $\mathscr{D}(T)$ belonging to the complex plane $t=0$. This curve is chosen such that all trajectories cross the plane $\Im(x)=0$ at the same time $T$.

and the solution $S_{0}\left(\mathbf{x}_{0}, t\right)$, the resulting function $S_{0}(\mathbf{x}, t)$ is the solution of the HJE (11) compatible with the initial condition (9), for all values of $\mathbf{x}$ and $t$, not only for $\mathbf{x} \in \gamma(t)$. Practically, the analytical extension can be made through an extrapolation scheme from complex $\gamma(t)$ to $\mathbb{R}^{d}$. This is a feasible way, not reported here.

(2) The alternative procedure is to use the extension theorem at the initial time, that is, to consider complex starting positions $\mathbf{x}_{0}$ for the complex trajectories. It amounts to extend analytically $S_{0}\left(\mathbf{x}_{0}, 0\right)$ into the complex domain. The relations (22)-(23) are still valid, taken as complex identities. It is necessary then to find out the starting point $\mathbf{x}_{0}$ such that the trajectory $\mathbf{x}\left(\mathbf{x}_{0}, t\right)$ crosses the surface $\mathfrak{I}(\mathbf{x})=0$ for a given time $t$, see Fig. 1(b) in the case $d=1$. The corresponding value of $S_{0}$ will provide $S_{0}(\mathbf{x}, t)$ with real $\mathbf{x}$ as looked for. This is the practical way we have adopted to sample the function $S_{0}(\mathbf{x}, t)$, the exact procedure will be given later.

As a partial conclusion, let us say that the different points discussed previously cover essentially the originality and the necessary concepts of the semiclassical method of complex trajectories that we propose.

\section{Gaussian initial wave packet}

The simplest choice of an initially localized wave packet with a corresponding analytic $\Phi$ function [cf. Eq. (9)] is the Gaussian wave packet reading:

$$
\psi_{\text {init }}(\mathbf{x})=\exp \left(i \mathbf{k}_{c} \cdot\left(\mathbf{x}-\mathbf{x}_{c}\right)-\frac{\left(\mathbf{x}-\mathbf{x}_{c}\right)^{2}}{a^{2}}\right) .
$$

$\mathbf{x}_{c}$ is the average value of position, $\mathbf{k}_{c}$ is the average wave number, $a$ is the width of the wave packet. Following our peculiar scheme (9)-(10), we have

$$
S_{0}(\mathbf{x}, 0)=\mathbf{p}_{c} \cdot\left(\mathbf{x}-\mathbf{x}_{c}\right)+i \frac{\left(\mathbf{x}-\mathbf{x}_{c}\right)^{2}}{\widetilde{a}^{2}},
$$

where $\mathbf{p}_{c}=\hbar \mathbf{k}_{c}$ and $\tilde{a}=a / \sqrt{\hbar}$. The final expression of the semiclassical initial wave function is

$$
\exp \left(i \frac{S_{0}(\mathbf{x}, 0)}{\hbar}\right)=\exp \left(i \frac{\mathbf{p}_{c}}{\hbar} \cdot\left(\mathbf{x}-\mathbf{x}_{c}\right)-\frac{\left(\mathbf{x}-\mathbf{x}_{c}\right)^{2}}{\hbar \widetilde{a}^{2}}\right),
$$

where $\mathbf{p}_{c}$ and $\tilde{a}$ are kept constant while $\hbar \rightarrow 0$. If $\mathbf{x}_{0}$ is the complex initial integration point, the initial conditions for the CEs, derived from Eq. (25) and Eqs. (22)-(23), read:

$$
\begin{aligned}
\mathbf{p}\left(\mathbf{x}_{0}, 0\right) & =\mathbf{p}_{c}+2 i \frac{\mathbf{x}_{0}-\mathbf{x}_{c}}{\widetilde{a}^{2}} \\
E_{0}\left(\mathbf{x}_{0}\right) & =H\left(\mathbf{x}_{0}, \mathbf{p}\left(\mathbf{x}_{0}, 0\right)\right) \\
& =\frac{1}{2 m}\left(\mathbf{p}_{c}+2 i \frac{\mathbf{x}_{0}-\mathbf{x}_{c}}{\widetilde{a}^{2}}\right)^{2}+V\left(\mathbf{x}_{0}\right) .
\end{aligned}
$$

One can notice that if $\mathbf{x}_{0}=\mathbf{x}_{c}$, then:

$$
\begin{aligned}
& S_{0}\left(\mathbf{x}_{c}, 0\right)=0, \\
& \mathbf{p}\left(\mathbf{x}_{c}, 0\right)=\mathbf{p}_{c} \text { is real, } \\
& E_{0}\left(\mathbf{x}_{c}\right)=\frac{\mathbf{p}_{c}^{2}}{2 m}+V\left(\mathbf{x}_{c}\right) \text { is real. }
\end{aligned}
$$

The initial conditions are thus real and since the CEs (19)(20) are real too, the corresponding complex trajectory is itself entirely real. If $\mathbf{x}_{0} \neq \mathbf{x}_{c}$, this is no longer the case. We will call this peculiar trajectory the real trajectory associated with the initial wave packet (24), it will be noted subsequently $\left(x_{t}, p_{t}\right)$.

Let us make now an important remark about the formal way our formalism leads to the semiclassical limit. Considering the initial wave function (26), we note that:

(1) As in the WKB method, the limit $\hbar \rightarrow 0$ causes the local wave length $2 \pi /\left(\left\|\mathbf{p}_{c}\right\| / \hbar\right)$ to tend to zero as $\hbar$. The classical momentum $\mathbf{p}_{c}=\hbar \mathbf{k}_{c}$ is a conserved quantity at the semiclassical limit.

(2) Unlike in the WKB method, the spatial extension of the wave packet is also bound to go to zero (in this case as $\sqrt{\hbar}$ ) because $\tilde{a}=a / \sqrt{\hbar}$ is the conserved quantity at the semiclassical limit, not the width $a$ itself as in the WKB formalism.

\section{First order}

The first order of the $\hbar$-expansion gives the equation satisfied by $S_{1}$ : 


$$
\frac{1}{m} \partial_{\mathbf{x}} S_{0} \cdot \partial_{\mathbf{x}} S_{1}+\partial_{t} S_{1}=\frac{i}{2 m} \nabla^{2} S_{0} .
$$

Thus, again, the PDE satisfied by $S_{1}$ is a single first order equation where $S_{0}$, previously determined, enters. The theory of characteristics can be used to solve this equation, in a simplified version, restricted to the configuration space, since Eq. (29) is linear in $S_{1}$. The CEs parametrized by time are the following:

$$
\begin{aligned}
& \frac{d x_{k}}{d t}=\frac{p_{k}(\mathbf{x}, t)}{m}, \\
& \frac{d S_{1}}{d t}=\frac{i}{2 m} \nabla^{2} S_{0}(\mathbf{x}, t) .
\end{aligned}
$$

Since the functions $p_{k}(\mathbf{x}, t)$ are calculated through the CEs (19)-(20) and Eq. (30) is the same as Eq. (20), the characteristics of $S_{1}$ are the canonical projection of the complex trajectories associated with $S_{0}$ on the configuration space (they remain nevertheless complex). This property will be of great help later. Notice finally that the arguments given in Section II B can be applied to $S_{1}$, provided it is analytic.

To conclude up to this point, let us say that we achieve the approximate solution, by means of a complex semiclassical theory, including the zeroth and first orders, of the problem of quantum wave packet propagation. The set of ODEs we have to consider is thus (19)-(20) to propagate the trajectories, (21) and (31) to derive the wave function, with the initial conditions (9)-(10) and (22)-(23), reading Eqs. (25) and (27)-(28) for an initial Gaussian wave. Let us note that an arbitrary $n$th order expansion can be carried out, leading to the following first order PDEs satisfied by each function $S_{n}$ :

$\frac{1}{m} \partial_{\mathbf{x}} S_{0} \cdot \partial_{\mathbf{x}} S_{n}+\partial_{t} S_{n}=\frac{i}{2 m} \nabla^{2} S_{n-1}-\frac{1}{2 m} \sum_{k=1}^{n-1} \partial_{\mathbf{x}} S_{k} \cdot \partial_{\mathbf{x}} S_{n-k}$.

These will be considered in Section III. Before describing examples, let us derive a new set of ODEs in order to propagate the second derivative of the action, a convenient tool for numerical calculations.

\section{E. Propagation of the second derivatives of the action}

We need to solve Eq. (29) satisfied by $S_{1}$. $S_{0}$ and its first order derivatives are calculated at the previous order by the HJE (11), solved by means of CEs. Nevertheless the Laplacian itself of $S_{0}$ does not result from these calculations if they are carried out numerically. A trick would consist of calculating it by means of a finite difference scheme (or a more sophisticated method derived from it), but this is tedious and does not offer sufficient accuracy. Rather, we would wish to propagate the quantity $\nabla^{2} S_{0}$ along the complex trajectories as well, in order to have at our disposal a value of $\nabla^{2} S_{0}$ at the same point as the other quantities and with the same accuracy.

Let us derive the set of equations satisfied by the second derivatives of $S_{0}$. As we shall see, we need to assume that $S_{0}$ is a well-defined function of $(\mathbf{x}, t)$, that is the characteristic curves do not cross caustics. The main idea is the following: the second derivatives of $S_{0}$ are $\partial_{k} p_{l}$, then, considering two neighboring trajectories, the infinitesimal difference of the momenta at the same time gives the matrix $\partial_{k} p_{l}$ whose trace is the Laplacian looked for.

Let us write these trajectories as $\mathbf{x}\left(\mathbf{x}_{0}, t\right)$ and $\mathbf{x}\left(\mathbf{x}_{0}\right.$ $\left.+\delta \mathbf{x}_{0}, t\right)=\mathbf{x}\left(\mathbf{x}_{0}, t\right)+\delta \mathbf{x}(t)$. Their canonical equations are the following, written as a first order expansion with respect to time:

$$
\begin{aligned}
& \mathbf{x}(t+d t)=\mathbf{x}(t)+\frac{\mathbf{p}(\mathbf{x}(t))}{m} d t, \\
& p_{l}(\mathbf{x}(t+d t))=p_{l}(\mathbf{x}(t))-\nabla V(\mathbf{x}(t)) d t, \\
& (\mathbf{x}+\delta \mathbf{x})(t+d t)=\mathbf{x}(t)+\delta \mathbf{x}(t)+\frac{\mathbf{p}((\mathbf{x}+\delta \mathbf{x})(t))}{m} d t, \\
& p_{l}((\mathbf{x}+\delta \mathbf{x})(t+d t)) \\
& \quad=p_{l}((\mathbf{x}+\delta \mathbf{x})(t))-\nabla V((\mathbf{x}+\delta \mathbf{x})(t)) d t .
\end{aligned}
$$

Expanding Eq. (36) with respect to $\delta \mathbf{x}$ and taking the difference with Eq. (34), one has:

$$
\begin{aligned}
& \frac{\partial p_{l}}{\partial x_{k}}(\mathbf{x}(t+d t)) \delta x_{k}(t+d t) \\
& \quad=\frac{\partial p_{l}}{\partial x_{k}}(\mathbf{x}(t)) \delta x_{k}(t)-\frac{\partial^{2} V}{\partial x_{l} \partial x_{k}}(\mathbf{x}(t)) \delta x_{k}(t) d t,
\end{aligned}
$$

where summation over repeated indices is assumed. The difference between Eqs. (33) and (35) leads to :

$$
\delta x_{k}(t+d t)=\delta x_{k}(t)+\frac{\partial p_{k}}{\partial x_{q}}(\mathbf{x}(t)) \frac{\delta x_{q}(t)}{m} d t .
$$

By substituting Eq. (38) into Eq. (37) and discarding third order terms, we derive a set of ODEs for the total time derivative - the derivative along the complex trajectories of the quantities $\partial_{k} p_{l}$ :

$$
\frac{d}{d t}\left(\frac{\partial p_{l}}{\partial x_{k}}\right)=-\frac{1}{m}\left(\frac{\partial p_{l}}{\partial x_{q}}\right)\left(\frac{\partial p_{q}}{\partial x_{k}}\right)-\frac{\partial^{2} V}{\partial x_{l} \partial x_{k}} .
$$

This equation is satisfied by the Jacobian of the function $\mathbf{x} \mapsto \mathbf{p}(\mathbf{x}, t)$ whose trace gives the Laplacian $\nabla^{2} S_{0}$ entering in the CE (31). In fact, it is not particular to the CTM, it can be derived to calculate the usual first order correction. ${ }^{20}$

Now, the set of ODEs (19)-(21), (31) and (39) makes a complete set of equations from a numerical point of view, in the sense that no extra operation is needed to integrate them. The total number of scalar equations is $(d+1)^{2}+1$ where $d$ is the number of degrees of freedom.

At this point, we have all the tools to compute analytically and numerically a propagated wave packet in the semiclassical theory of complex trajectories provided that the functions involved have analytical dependence with the position. This is the aim of the next subsection.

\section{F. Analyticity, caustics, unitarity and comparison with the Gaussian wave packet propagation theory}

As stressed before, previous derivations make sense only if the functions $S_{0}$ and $S_{1}$, from which the semiclassical 
wave function is built, depend analytically on the position variables for every time. We can show easily in the case of the 1D Schrödinger equation, which condition is required for this analycity property to be valid.

Let us call $G(z, t)$, any of the functions $S_{0}(z, t)$ and $S_{1}(z, t)$ of the complex variable $z$ and the real time variable $t$. Assuming $G$ analytical in $z$ at some time $t$, an equivalent property is the following: if $\Gamma$ is any closed contour in the complex plane, we have

$$
\forall \Gamma, \quad I(t)=\oint_{\Gamma} G(z, t) d z=0 .
$$

We calculate then such a contour integral at time $t+d t$. Expanding with respect to time and applying Equation (40), we get

$$
I(t+d t)=d t \oint_{\Gamma} \frac{\partial G}{\partial t}(z, t) d z .
$$

In the case $G=S_{0}$, from Eq. (11), we have

$$
I(t+d t)=-d t\left(\oint_{\Gamma} \frac{1}{2 m}\left(\frac{\partial S_{0}}{\partial z}(z, t)\right)^{2} d z+\oint_{\Gamma} V(z) d z\right) .
$$

$S_{0}(z, t)$ analytic implies $\left(\partial S_{0} / \partial z\right)(z, t)$ is too, and so the condition of analyticity of $S_{0}(z, t+d t)$ reads:

$$
\forall \Gamma, \quad I(t+d t)=-d t \oint_{\Gamma} V(z) d z=0,
$$

that is, the potential $V(z)$ is analytic. In the case $G=S_{1}$, from Eq. (29), we have

$$
\begin{aligned}
I(t+d t)= & d t\left(\oint_{\Gamma} \frac{i}{2 m}\left(\frac{\partial^{2} S_{0}}{\partial z^{2}}(z, t)\right) d z\right. \\
& \left.-\oint_{\Gamma} \frac{1}{m}\left(\frac{\partial S_{0}}{\partial z}(z, t)\right)\left(\frac{\partial S_{1}}{\partial z}(z, t)\right) d z\right) .
\end{aligned}
$$

Thus $S_{1}(z, t+d t)$ is analytic since $S_{0}(z, t)$ and $S_{1}(z, t)$ are, from the hypothesis (40). Finally, provided that the potential $V(z)$ is analytic as well as the initial function $S_{0}(z, 0)$ $\left(S_{1}(z, 0)\right.$ is as it is equal to zero), the functions of interest $S_{0}(z, t)$ and $S_{1}(z, t)$ are analytic for every time.

The theory stated previously, like any other semiclassical theories, can entail singularities (caustics). These are caused geometrically due to the fact that the set of points described by the characteristics in the complete phase space (a Lagrangian manifold of dimension $d+1$ ) does not have a one-to-one canonical projection onto the complete configuration space (position and time). Whereas the $S_{0}$ function is a well-defined function on this Lagrangian manifold, it is no more uniquely defined on the initial complete configuration space. From an analytical point of view, ${ }^{21}$ it causes the divergence of one or more matrix elements of the Jacobian of the function $\mathbf{p}(\mathbf{x}, t)$. In the one dimensional case, and if the HJE is the usual one (11), $\partial p / \partial x$ diverges at the intersection with a caustic. Numerically, it causes the failure of the inte- gration algorithm and thus provides a way of detecting these intersections. This property will be exploited further on numerical examples.

Another question that can be asked about our complex method is whether it is unitary or not. The WKB method restricted to the first order is. In our case, by writing the semiclassical wave function (5) restricted to the first order approximation, we can show

$$
\begin{aligned}
& \frac{d}{d t} \int|\psi(\mathbf{x}, t)|^{2} d \mathbf{x} \\
& \quad=\frac{\hbar^{2}}{2 m} \int\left(\nabla^{2}\left(\frac{S_{1}}{\hbar}\right)+i \hbar\left(\frac{\nabla S_{1}}{\hbar}\right)^{2}+\text { c.c. }\right)|\psi(\mathbf{x}, t)|^{2} d \mathbf{x},
\end{aligned}
$$

denoting "complex conjugate" as "c.c." This shows that our method is approximately unitary, consistent with the order of the approximation considered, that is the error is of one order higher in $\hbar$ than the order of the last term taken into account (the form of Eq. (45) suggests that it might be true at all orders). The last equation results from the following identity, that can be easily derived from the HJE (11) and Eq. (29):

$$
\begin{aligned}
\frac{\partial}{\partial t} \exp \left(i\left(S_{0}-S_{0}^{*}\right) / \hbar+i\left(S_{1}-S_{1}^{*}\right)\right) \\
=-\frac{1}{2 m} \nabla \cdot\left[\left(\nabla S_{0}+\nabla S_{0}^{*}+\hbar\left(\nabla S_{1}+\nabla S_{1}^{*}\right)\right)\right. \\
\left.\quad \times \exp \left(i\left(S_{0}-S_{0}^{*}\right) / \hbar+i\left(S_{1}-S_{1}^{*}\right)\right)\right] \\
+\frac{\hbar}{2 m}\left(\nabla^{2} S_{1}+i\left(\nabla S_{1}\right)^{2}+\text { c.c. }\right) \\
\quad \times \exp \left(i\left(S_{0}-S_{0}^{*}\right) / \hbar+i\left(S_{1}-S_{1}^{*}\right)\right),
\end{aligned}
$$

and under the hypothesis that the wave function is vanishingly small at infinity.

As a last remark, we can compare the formalism of the Gaussian wave packet propagation method ${ }^{15}$ (GWPPM) of E. J. Heller to ours. It is easy to show that by expanding the generalized action $S_{0}$ as a second order expansion in position around the point $x_{t}$ reached by the real trajectory at time $t$, restricting the expansion to the first order and using the equations of propagation derived before, our method is equivalent to the GWPPM. If $S_{0}$ is not restricted to the preceding expansion, we will see in the numerical examples given afterwards that the complex trajectory method is not equivalent to the GWPPM.

We have now entirely described the principle of the complex trajectory method (CTM), we will now apply it to several potentials. In each case, the comparison between the exact and the approximated wave packets is made. The results given by the WKB method or the GWPPM will also be mentioned.

\section{SOLUBLE PROBLEMS}

This section is devoted to the analytical propagation of one dimensional quantum wave packets at the semiclassical 
limit by means of the complex trajectory method. Three potentials will be reviewed: the flat, linear and harmonic potentials, the first two being treated together.

\section{A. Linear and flat potentials}

The potential we consider here is

$V(x)=\alpha x$,

where $\alpha$ is a constant, $\alpha=0$ giving the case of the flat potential. The Hamilton-Jacobi equation (11) reads

$$
J=\frac{1}{2 m}\left(\partial_{x} S_{0}\right)^{2}+\alpha x+\partial_{t} S_{0} .
$$

It is solved by means of the corresponding characteristic equations (19)-(21), reading here as

$$
\begin{aligned}
& \frac{d x}{d t}=\frac{p}{m}, \\
& \frac{d p}{d t}=-\alpha, \\
& \frac{d S_{0}}{d t}=\frac{p^{2}}{m}-E_{0} .
\end{aligned}
$$

The initial wave packet is taken as a one dimensional Gaussian centered around zero:

$$
\psi(x, 0)=\exp \left(i \frac{p_{c} x}{\hbar}-\frac{x^{2}}{a^{2}}\right)=\exp \left(i S_{0}(x, 0) / \hbar\right) .
$$

From Eqs. (25), (27)-(28) and denoting $x_{0}$ as a complex starting point, the initial conditions are

$$
\begin{aligned}
& S_{0}\left(x_{0}, 0\right)=p_{c} x_{0}+i \frac{x_{0}^{2}}{\widetilde{a}^{2}}, \\
& p\left(x_{0}, 0\right)=\left(\frac{\partial S_{0}(x, 0)}{\partial x}\right)_{x_{0}}=p_{c}+2 i \frac{x_{0}}{\widetilde{a}^{2}}, \\
& E_{0}\left(x_{0}\right)=\frac{\left(p\left(x_{0}, 0\right)\right)^{2}}{2 m}+\alpha x_{0}=\frac{1}{2 m}\left(p_{c}+2 i \frac{x_{0}}{\widetilde{a}^{2}}\right)^{2}+\alpha x_{0} .
\end{aligned}
$$

The set of equations (49)-(55) is all that is required to compute $S_{0}(x, t)$.

From Eqs. (50) and (54), we have the value of the momentum $p$ at time $t$ along the trajectory starting at $x_{0}$ :

$$
p\left(x_{0}, t\right)=-\alpha t+p_{c}+\frac{2 i x_{0}}{\widetilde{a}^{2}} .
$$

This allows the immediate integration of Eq. (49), giving the position $x$ along the same trajectory at the same time:

$$
x\left(x_{0}, t\right)=\frac{-\alpha t^{2}}{2 m}+\frac{p_{c} t}{m}+x_{0}\left(1+\frac{2 i t}{m \widetilde{a}^{2}}\right) .
$$

From Eq. (51), substituting $p$ by Eq. (56) and using initial condition (55), we derive the value of $S_{0}$ along the trajectory:

$$
\begin{aligned}
S_{0}\left(x_{0}, t\right)= & \frac{\alpha^{2} t^{3}}{3 m}-\frac{\alpha t^{2} p_{c}}{m}+\frac{p_{c}^{2} t}{2 m} x_{0}\left(p_{c}-\alpha t\right)\left(1+\frac{2 i t}{m \widetilde{a}^{2}}\right) \\
& +\frac{i x_{0}^{2}}{\widetilde{a}^{2}}\left(1+\frac{2 i t}{m \widetilde{a}^{2}}\right) .
\end{aligned}
$$

The elimination of the starting point $x_{0}$ between $x\left(x_{0}, t\right)(57)$ and $S_{0}\left(x_{0}, t\right)(58)$ allows the action $S_{0}(x, t)$ to be derived as a function of its natural variables $(x, t)$ :

$$
\begin{aligned}
S_{0}(x, t)= & \left(\frac{\alpha^{2} t^{3}}{3 m}-\frac{\alpha t^{2} p_{c}}{m}+\frac{p_{c}^{2} t}{2 m}\right)+p_{t}\left(x-x_{t}\right) \\
+ & i \frac{\left(x-x_{t}\right)^{2}}{\widetilde{a}^{2}+\frac{2 i t}{m}},
\end{aligned}
$$

$\left(x_{t}, p_{t}\right)$ is the real trajectory at time $t$ associated with the initial wave packet (52), for which $x_{0}$ is equal to its center, 0 . It reads:

$$
x_{t}=\frac{-\alpha t^{2}}{2 m}+\frac{p_{c} t}{m}, \quad p_{t}=p_{c}-\alpha t .
$$

From Eq. (29) the first order correction satisfies:

$$
\frac{1}{m} \partial_{x} S_{0} \partial_{x} S_{1}+\partial_{t} S_{1}=\frac{i}{2 m} \partial_{x^{2}}^{2} S_{0} .
$$

The resulting characteristic equations are

$$
\begin{aligned}
& \frac{d x}{d t}=\frac{p}{m}, \\
& \frac{d S_{1}}{d t}=\frac{i}{2 m} \partial_{x^{2}}^{2} S_{0} .
\end{aligned}
$$

Since $\partial_{x^{2}}^{2} S_{0}$, calculated from Eq. (59), is independent of $x$ :

$$
\partial_{x^{2}}^{2} S_{0}(x, t)=\frac{2 i}{\widetilde{a}^{2}+\frac{2 i t}{m}},
$$

Eq. (62) is not needed. $S_{1}(x, t)$ is simply calculated by integration of Eq. (63). Using Eq. (64), we have

$$
S_{1}(x, t)=\frac{i}{2} \ln \left(1+\frac{2 i t}{m \widetilde{a}^{2}}\right) .
$$

Finally, $e^{i\left(S_{0} / \hbar+S_{1}\right)}$ gives the semiclassical wave function obtained by the CTM up to first order. Restoring the $\hbar \mathrm{s}$, we obtain:

$$
\begin{aligned}
\psi_{c l}(x, t)= & \frac{1}{\left(1+\frac{2 i t \hbar}{m a^{2}}\right)^{1 / 2}} \exp \left(i\left(\frac{\alpha^{2} t^{3}}{3 m \hbar}-\frac{\alpha t^{2} p_{c}}{m \hbar}+\frac{p_{c}^{2} t}{2 m \hbar}\right)\right. \\
& \left.+i \frac{p_{t}}{\hbar}\left(x-x_{t}\right)-\frac{\left(x-x_{t}\right)^{2}}{a^{2}+\frac{2 i t \hbar}{m}}\right)
\end{aligned}
$$

We can recognize here the exact solution of the Schrödinger equation with the initial condition (52) in the cases $\alpha=0^{22}$ 
and $\alpha \neq 0^{23}$ that is, in the cases of the flat and linear potentials. In these cases, the WKB approximation would have led to a fixed width wave packet. Here, the spreading of the wave packet is exactly predicted. One notices that, in the CTM, the preexponential factor appears as the quantum correction to the purely classical, i.e., zeroth order approximation, unlike with the WKB method. Considering Eq. (32) of the higher order terms, it is easy to show that $S_{1}$ independent of $x$ implies that all other $S_{j}$ are equal to zero, the previous result is thus the approximation of the CTM for all orders.

In the case of the flat potential, making $\alpha=0$, we have the wave function

$$
\psi_{c l}=\frac{e^{i \frac{p_{c} x-E t}{\hbar}}}{\sqrt{1+\frac{2 i t \hbar}{m a^{2}}}} \exp \left(-\frac{\left(x-x_{t}\right)^{2}}{a^{2}+\frac{2 i t \hbar}{m}}\right),
$$

where $E=p_{c}^{2} / 2 m$ and $x_{t}=p_{c} t / m$. Let us make the following comments:

(1) Making $\hbar \rightarrow 0$ in the exact expression of the propagated wave packet (67) with $a$ constant, gives a different expression from the exact one which is the result of the WKB method restricted to the first order (a fixed width wave packet). On the contrary, making $\hbar \rightarrow 0$ with $\tilde{a}=a / \sqrt{\hbar}$ remaining constant, i.e., the width $a$ going to zero as $\sqrt{\hbar}$, leaves the expression of the wave packet (67) formally unaltered. As stated in Section II C, this is precisely what is done in the CTM, explaining its success in this case. The same remark is true for all other analytical results, cf. Eqs. (66) and (79).

(2) Here, the WKB wave function can be explicitly computed to all orders in $\hbar$, expanding the amplitude $A$ as a power series in $\hbar$. The result is a series which is the Taylor expansion of the exact result (67) with respect to the dimensionless parameter $2 i \hbar t / m a^{2}$, with a radius of convergence equal to 1 due to the singularity of the wave function (67). This gives a finite time limit $m a^{2} / 2 \hbar$ for the convergence. Straight time integration and summation of the series even at all orders cannot break this time barrier: an analytic continuation is needed. Using the CTM we derive an exact finite order expansion, that is, with an infinite radius of convergence. This clearly means then that adopting the unusual initial conditions (9) and (10) instead of Eqs. (6)-(8) has a very important consequence on the expansion itself.

\section{B. Harmonic potential}

We now consider the harmonic potential:

$$
V(x)=\frac{1}{2} m \omega^{2} x^{2} .
$$

If the initial wave packet is taken as previously (52), then the previous initial conditions (53) and (54) hold. The characteristic equations derived from the HJE read here as:

$$
\frac{d}{d t}(\omega x)=\omega \frac{p}{m},
$$

$$
\begin{aligned}
& \frac{d}{d t}\left(\frac{p}{m}\right)=-\omega(\omega x), \\
& \frac{d S_{0}}{d t}=\frac{p^{2}}{m}-E_{0} .
\end{aligned}
$$

With the same initial conditions as previously (53)-(55), the resolution of Eqs. (69) and (70) of the harmonic oscillator in the complex phase space leads to

$$
\begin{aligned}
x\left(x_{0}, t\right)= & x_{0} \cos (\omega t)+\frac{p\left(x_{0}, 0\right)}{m \omega} \sin (\omega t) \\
= & x_{0}\left(\cos (\omega t)+\frac{2 i}{m \omega \widetilde{a}^{2}} \sin (\omega t)\right) \\
& +\frac{p_{c}}{m \omega} \sin (\omega t), \\
p\left(x_{0}, t\right)= & p\left(x_{0}, 0\right) \cos (\omega t)-m \omega x_{0} \sin (\omega t) \\
= & x_{0}\left(\frac{2 i}{\widetilde{a}^{2}} \cos (\omega t)-m \omega \sin (\omega t)\right)+p_{c} \cos (\omega t) .
\end{aligned}
$$

The usual procedure would consist of substituting Eq. (73) into Eq. (71), then integrating and eliminating $x_{0}$ between the result and Eq. (72). We prefer here to eliminate $x_{0}$ between Eq. (72) and Eq. (73), yielding the momentum as a function of its natural variables $(x, t)$, and to integrate with respect to $x$, using the definition $p=\partial_{x} S_{0}$. This leads to:

$$
\begin{aligned}
S_{0}(x, t)= & \frac{m \omega}{2}\left(\frac{-\sin (\omega t)+\frac{2 i}{m \omega \widetilde{a}^{2}} \cos (\omega t)}{\cos (\omega t)+\frac{2 i}{m \omega \widetilde{a}^{2}} \sin (\omega t)}\right)\left(x-x_{t}\right)^{2} \\
& +p_{t}\left(x-x_{t}\right)+K(t)
\end{aligned}
$$

where the real trajectory $\left(x_{t}, p_{t}\right)$ is

$$
x_{t}=\frac{p_{c}}{m \omega} \sin (\omega t), \quad p_{t}=p_{c} \cos (\omega t)
$$

$K(t)$ being an unknown function of time. To determine $K(t)$, we integrate Eq. (71) along the real trajectory starting at $x_{0}=0$. We have then

$$
K(t)=S_{0}\left(x_{t}, t\right)=\frac{p_{c}^{2}}{2 m \omega} \sin (\omega t) \cos (\omega t) .
$$

The first order correction satisfies Eqs. (62)-(63) giving:

$$
\frac{d S_{1}}{d t}=\frac{i}{2 m} \frac{\partial^{2}}{\partial x^{2}} S_{0}=\frac{i \omega}{2}\left(\frac{-\sin (\omega t)+\frac{2 i}{m \omega \tilde{a}^{2}} \cos (\omega t)}{\cos (\omega t)+\frac{2 i}{m \omega \tilde{a}^{2}} \sin (\omega t)}\right) .
$$

This equation being $x$ independent, its straightforward integration gives the function $S_{1}(x, t)$ : 


$$
S_{1}(x, t)=\frac{i}{2} \ln \left(\cos (\omega t)+\frac{2 i}{m \omega \tilde{a}^{2}} \sin (\omega t)\right) .
$$

From Eqs. (74), (76) and (78), we obtain the semiclassical wave function by means of the CTM:

$$
\begin{aligned}
\psi_{c l}= & \frac{1}{\sqrt{R(t)}} \exp \left(\frac{i m}{2 \hbar} \frac{R^{\prime}(t)}{R(t)}\left(x-x_{t}\right)^{2}+\frac{i p_{t}}{\hbar}\left(x-x_{t}\right)\right. \\
& \left.+\frac{i p_{c}^{2}}{2 m \omega \hbar} \sin (\omega t) \cos (\omega t)\right),
\end{aligned}
$$

where $R(t)=\cos (\omega t)+\left(2 i \hbar / m \omega a^{2}\right) \sin (\omega t)$ and $R^{\prime}$ is its time derivative. One can check that $\psi_{c l}$ is exactly the quantum wave packet propagated in the harmonic potential that is initially given by Eq. (52). ${ }^{22}$ For the same reason as in the previous case, this result is the prediction of the CTM for all orders.

The comparison with the WKB method is interesting from the point of view of the study of caustics. The result of this method can be obtained from the previous calculations by taking the limit $a \rightarrow \infty$ in trajectory Equations (72)-(73), making the complex contributions equal to zero. It is easy to show then that the initial condition (52) makes the point $\left(t_{f}\right.$ $\left.=\pi / 2 \omega, x_{f}=p_{c} / m \omega\right)$ a focal point - the property of isochronism in the permuted variables $(p, x)$ - causing the divergence of $\partial_{x} p=-i \omega \tan (\omega t) / 2$ [from Eq. (77)] at that point. In the complex trajectory treatment, no such annoying feature is encountered. Geometrically, no trajectory crosses any other in the complete complex configuration space because $x_{0}$ can be eliminated for any real times $t$ between Eqs. (72) and (73) (in the WKB approach, $i / \widetilde{a}^{2}=0$ prevents such elimination). Analytically, from Eq. (77), we can easily conclude that $\partial_{x} p$ never diverges for any real times $t$.

Note that here a divergence occurs in the Van-Vleck propagator formula too because it contains the WKB value of $\partial_{x} p$; this is formally removed by the integration with an initial Gaussian wave packet.

As a partial conclusion, the following points can be noted from the analytical calculations carried out within the framework of the complex trajectory method:

(1) The CTM is as accurate as the Van-Vleck propagator and much more accurate than the WKB method.

(2) No caustics are encountered, unlike for the real semiclassical theories.

These points will be reexamined and discussed on numerical examples. Notice that, in the three previous cases, since the propagated wave packets remain Gaussian, the GWPPM $^{15}$ gives the exact result too.

\section{NUMERICAL PROPAGATIONS}

\section{A. Methodology}

In the soluble examples, the complex nature of the trajectories underneath is, in a way, nearly hidden because the algebraic properties of complex or real numbers are the same, so the analytical extension on which the calculations rely is automatically made. Stated otherwise, the formalism can be used blindly without caring about each of the trajectories. This is no longer the case in classically nonsoluble potentials where numerical trajectory calculations are required. We have used the second procedure described in Section II B. It consists in starting from a complex position in order to reach a real position, obtaining directly a physical value of $S_{0}$ and $S_{1}$. It requires the determination of the starting position $\mathbf{x}_{\mathbf{0}}$ of the complex trajectory reaching a given real position $\mathbf{q}$ for a given time $t$. This is a two-point boundary value problem that amounts to finding the zero of the following function:

$$
\mathbf{x}_{0} \mapsto \mathbf{x}\left(\mathbf{x}_{0}, t\right)-\mathbf{q},
$$

where $\mathbf{x}\left(\mathbf{x}_{0}, t\right)$ is the complex trajectory starting from $\mathbf{x}_{0}$. The Newton's method will be used, evaluating numerically the Jacobian of the function (80). The real trajectory corresponding to the initial Gaussian wave packet gives the zero, i.e., $\mathbf{x}_{c}$, of the function (80) if $\mathbf{q}$ is chosen as $\mathbf{x}_{t}$, which is the position reached by the real trajectory at time $t$. Then, for the same time $t$ and a neighboring position $\mathbf{q}$ of $\mathbf{x}_{t}, \mathbf{x}_{c}$ can be chosen as the starting point in the Newton's procedure. Such a scheme is repeated step by step to calculate the entire wave packet at time $t$.

The quantum or exact propagated wave packet is simply calculated by means of the time and space discretization of the time translation operator under the well-known Cayley's form. $^{24}$

\section{B. Wall of potential}

Here we will study numerically by means of the complex trajectory method the propagation of a wave packet in a potential chosen in order that the corresponding classical particle bounces against a wall. The potential considered is quartic:

$$
V(x)=\left(x^{4}-400 x^{2}\right) 1.25 \times 10^{-4},
$$

with units such as $\hbar=m=1$. The initial wave function is

$$
\psi(x, 0)=\exp \left(i k x-\frac{x^{2}}{a^{2}}\right)
$$

where the average wave number and the width of the potential are, respectively, $k=5$ and $a=1$. In Fig. 2(a), the potential is plotted as a function of $x$, the average energy of the wave packet is shown by a dotted line. In Fig. 2(b), the real trajectory associated with the initial wave packet (82) is plotted as a function of time, indicating roughly the mean position of the propagated wave packet.

The wave packets have been computed by means of the complex trajectory method and quantally, at successive times, and compared. The square modulus of the wave function is plotted as a function of $x$ for different times, see Fig. 3(a), for times before the bounce and, Fig. 3(b), for times afterwards.

Figure 3(a) shows the excellent accuracy of the CTM for the entire wave packet, as long as it has not touched the potential wall. The wave packet at time $t=4$, very well approximated semiclassically, is clearly not Gaussian. The 

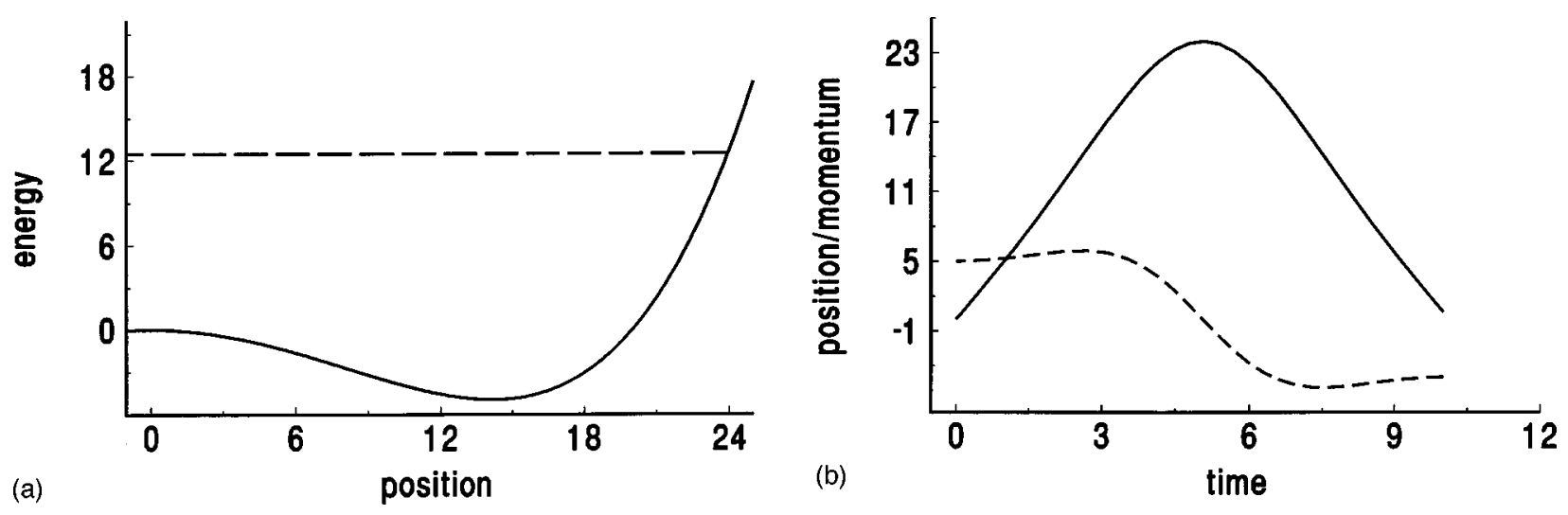

FIG. 2. On the left (a), the potentiel $V(x)$ (solid) of Section IV B is plotted with the mean energy of the wave packet, or the energy of its real trajectory (dashed line). On the right (b), the position (solid) and the momentum (dashed) of the real trajectory, are plotted as a function of time.

GWPPM would have predicted a Gaussian wave packet. This proves thus that the CTM is not equivalent to the GWPPM.

When the wave packet reaches the turning point, its mean momentum goes toward zero, that is, the classical regime condition is no longer valid. A natural discrepancy is thus observed between the semiclassical wave packet and the exact wave packet which displays oscillations due to interference between the forward and backward parts of the wave packet, cf. Figs. 3(a) and 3(b).

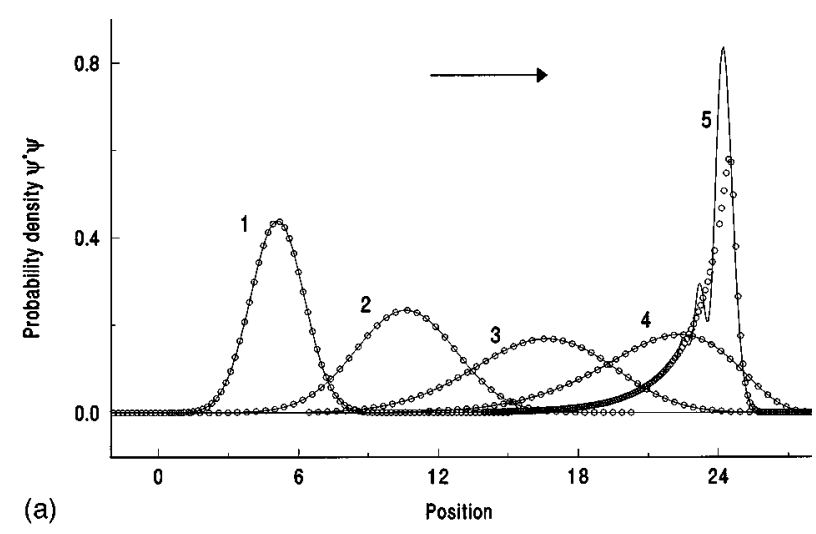

(a)

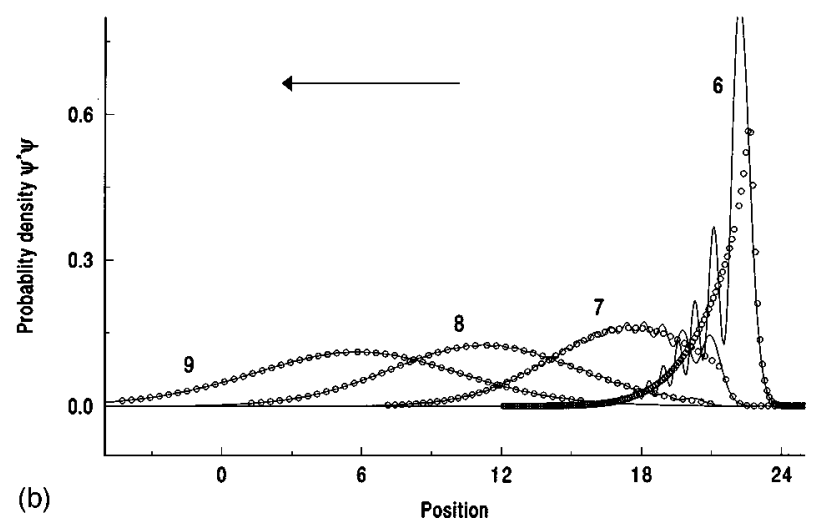

FIG. 3. The result of the quantum (solid) and semiclassical CTM (circles) propagations in the potential of Section IV B, at several times, indicated near the corresponding curve. (a) and (b) correspond respectively to times before and after the real trajectory reaches the turning point. Notice the remarkable accuracy of the CTM even after the wave packet has been reflected by the wall of potential.
Figure 3(b) shows nevertheless two interesting facts:

(1) As the wave packet leaves the neighborhood of the turning point, the semiclassical prediction recovers its accuracy for the entire wave packet.

(2) No singularity (caustic) has been encountered during the integration of the equations of the CTM whereas the usual real theories entail such a singularity due to the turning point.

We think that these appealing features, that can be noticed on the soluble examples too, give the complex trajectory method its main interest. We can sketch the following explanations.

(1) Despite the fact that the trajectories involved to build the wave packet after the bounce are "singular" from the semiclassical point of view, i.e., the momentum approaches zero around the turning point, the semiclassical prediction remains accurate. Particularly, the real trajectory runs exactly through the turning point and reaches the center of the wave packet. The integration with respect to time of the characteristic equations of $S_{0}$ and $S_{1}$ along a peculiar trajectory in the phase space, is an integral on the time line that can be extended in the complex domain. As such, it can be carried out along any deformed path, assuming analyticity with respect to time, avoiding the turning point. This works thus only once the wave packet has been entirely reflected backwards, not when it is concentrated near the turning point. This is coherent then with the error observed here and the recovery of the accuracy afterward.

(2) Unlike in the WKB treatment, no singularity stops the integration of the set of characteristic equations in the CTM, proving that no caustics are crossed. This means that the poles of the functions of time $\left(\partial_{x} p\right)^{-1}\left(x\left(x_{0}, t\right), t\right)$ that are isolated points, are not on the real axis but in the complex domain whereas in the WKB treatment, they lie on the real axis. We can interpret this fact by noticing that we work within a wider configuration space since it is complex (dimension 2). We can thus expect that this increase of available space might allow us to avoid the emergence of caustics, at least in the cases studied, and to reduce them in general. Using the Van-Vleck formula, the crossing with the caustics leads to the addition of a phase shift and prevents 


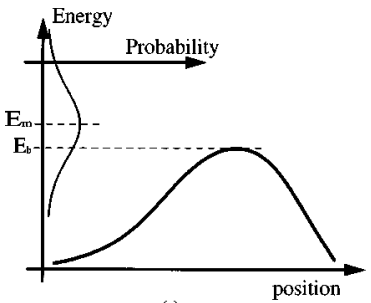

(t)

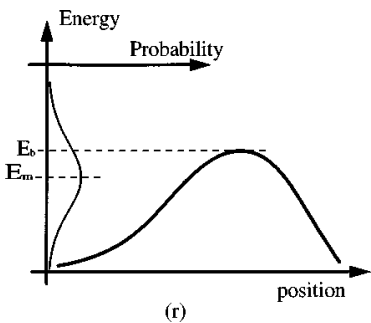

FIG. 4. The potential barrier is plotted as a function of the position and the wave packet, in energy representation, is plotted as a probability density as a function of the energy. Two cases are distinguished, (t) and (r), depending on whether the mean energy of the wave packet is respectively above or below the top of the barrier. In all the cases considered, we assume that the initial energy is quantally distributed above and below $E_{b}$.

one from calculating the Van-Vleck-Pauli-Morette determinant by propagation, forcing one to use a numerical derivative.

We can conclude that the very complex nature of the working space in the CTM seems to be responsible for the appealing property of reducing the singularities of our semiclassical treatment.

As final remarks, let us add that the comparison was made at times $t=4$ and $t=9$ between the real parts of the semiclassical and the exact wave functions on one hand, and between the imaginary ones, on the other hand. This leads to a very accurate approximation, similar to the ones observed on moduli. From Fig. 3, it is clear that the CTM works in the region not allowed classically, that is, there are complex trajectories reaching this region. Anyway, the analytical extension theorem would allow one to find the value of the wave function predicted by the CTM anywhere from the image of any segment of the complex domain, cf. Section II B. The bounce of a wave packet has been studied for other polynomial potentials as well as an $\exp (x)$-type potential. In these cases, as in the previous one, the same regularity and accuracy of the CTM is observed, except when the wave packet is in the neighborhood of the turning point.

\section{Crossing a potential barrier}

In this section, we will study the wave packet propagation in a more "difficult" case from the semiclassical point of view, in the sense that a wave packet splitting is expected to occur. To do this, we will consider wave packets "colliding' with a potential barrier, see Figure 4, that is potential curves bent toward negative energies.

The wave packet, in energy representation, is dispatched around the energy $E_{b}$ maximum of the barrier. Two cases may be then distinguished:

(t) transmit, the mean value of the energy of the wave packet $E_{m}$ is above the barrier maximum $E_{b}$. Classically, a particle of initial energy $E_{m}$ or the real trajectory associated with the initial wave packet, goes above the barrier. Quantally, the wave packet is essentially transmitted, but a reflected wave packet is expected to appear.

(r) reflect, the mean value of the energy of the wave packet $E_{m}$ is below the barrier maximum $E_{b}$. In this case, the real trajectory encounters a turning point while the quantum wave packet is expected to split.

In both these situations, two phenomena of a quantal nature arise:

(1) the tunneling effect for energies below the energy barrier $E_{b}$

(2) a less well-known effect: the bounce over a potential barrier ${ }^{9,10}$ for energies just above the energy barrier $E_{b}$.

We will study the complex trajectory method in this context on two examples.

\section{Quadratic potential}

Our aim is to study the semiclassical propagation on the potential $V(x)=-\frac{1}{2} m \omega x^{2}$, in the $(\mathrm{t})$ and $(\mathrm{r})$ cases. This is the "reversed" harmonic potential, so that, in fact, an analytical solution of this problem can be given. The exact solution given by the CTM can be found in Section III B, formula (79) replacing trigonometric functions " $\sin$ " and "cos" by their hyperbolic counterparts "sinh" and "cosh", respectively, and by replacing the minus sign preceding the "sin", functions by a plus sign. We can check that we get in this way the exact solution of the Schrödinger equation.

The position of the maximum follows the real trajectories associated with the initial wave packet, that is, it crosses the barrier in the (t) case and is reflected backward in the (r) case. The splitting of the wave packet is not observed but a fast exponential spreading of the wave packets occurs, as $\exp (\omega t / 2)$, accounting for the transmitted and reflected parts of the wave packets.

\section{Quasi-quartic potential}

The following potential is considered:

$$
V(x)=\left(-\frac{x^{4}}{10^{4}}+x\right) e^{-(x-5)^{2} / 1000},
$$

and is plotted in Fig. 5(a). It as been constructed so as to have a linear slope around zero cut by a steep part in order to observe the splitting of the wave packet. The exponential factor allows the avoidance of the infinite decrease of the potential that would prevent one from making a quantum simulation. The initial wave packet is chosen as

$$
\psi=\exp \left(i k\left(x-x_{c}\right)-\left(x-x_{c}\right)^{2}\right)
$$

with $x_{c}=5$. Two simulations were carried out for the (t) and (r) cases mentioned before.

(1) $(\mathrm{t}): k=3.2, E_{m} \simeq k^{2} / 2+V\left(x_{c}\right)=10.06>E_{b}=9.52$,

(2) (r): $k=2.8, E_{m} \simeq k^{2} / 2+V\left(x_{c}\right)=8.86<E_{b}=9.52$.

The corresponding real trajectories are plotted in Fig. 5(b). The mean energies $E_{m}$ are chosen quite close to the barrier energy $E_{b}$ in order that, in the $(\mathrm{t})$ case, the reflected wave is not negligible relative to the transmitted one, and vice versa in the (r) case.

The results are represented in Figures 6(a) and 6(b), corresponding to the $(\mathrm{t})$ and $(\mathrm{r})$ cases, respectively. The probability density is plotted as a function of the position at several times. As long as the wave packet remains Gaussian, the semiclassical prediction using the CTM is very accurate, as in the case treated before. Only one wave packet of this type 

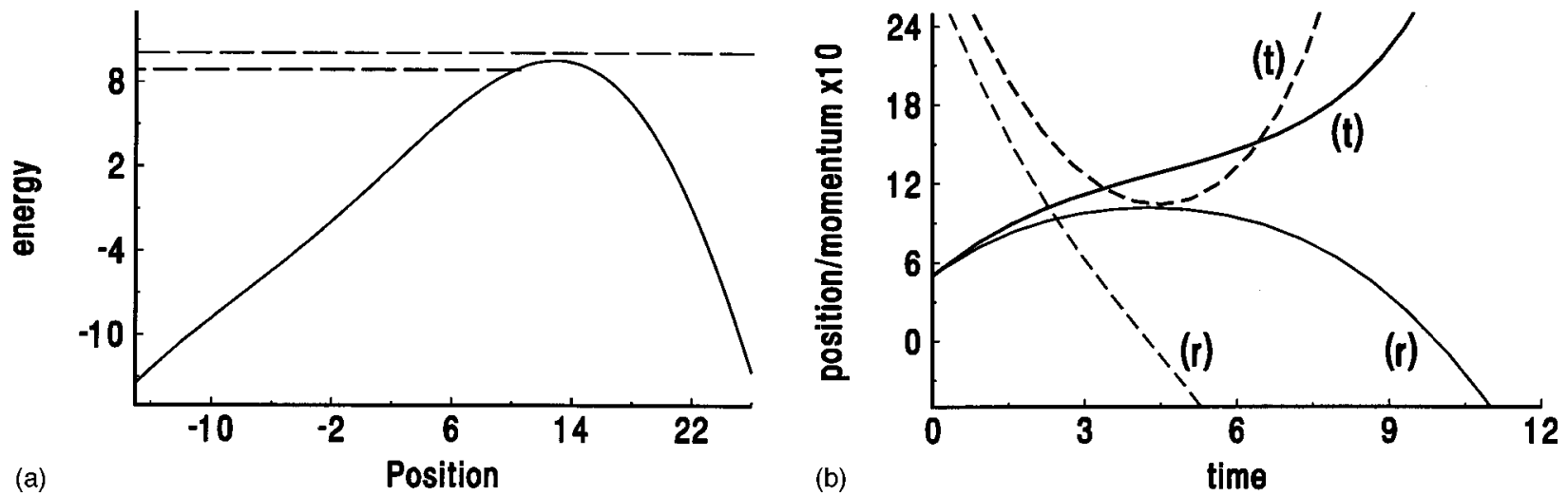

FIG. 5. On the left (a), the potentiel $V(x)$ (solid) of Section IV C 2 is plotted in the vicinity of the top of the barrier, together with the mean energy of the wave packet, or the energy of its real trajectory, in a dashed line. Both (t) and (r) cases are shown, corresponding to the two different initial wave packets, see the text. On the right (b), the position (solid) and the momentum (dashed) of the real trajectories, are plotted as a function of time, in the (t) and (r) cases.

is represented in Figures 6(a) and 6(b), corresponding to time $t=4$. It is remarkable that, at this time [see Fig. 5(b)], the classical particle following the real trajectory is either very near the top of the barrier, case $(t)$, or in the vicinity of the turning point, case (r). The accuracy observed is thus quite surprising since, from the previous case and particularly in the (r) case, we would have expected the failure of the CTM prediction around that singular point.

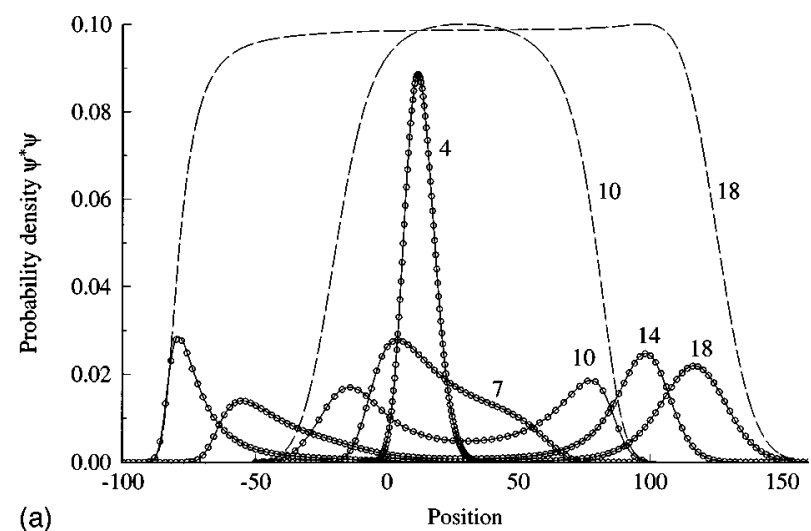

(a)

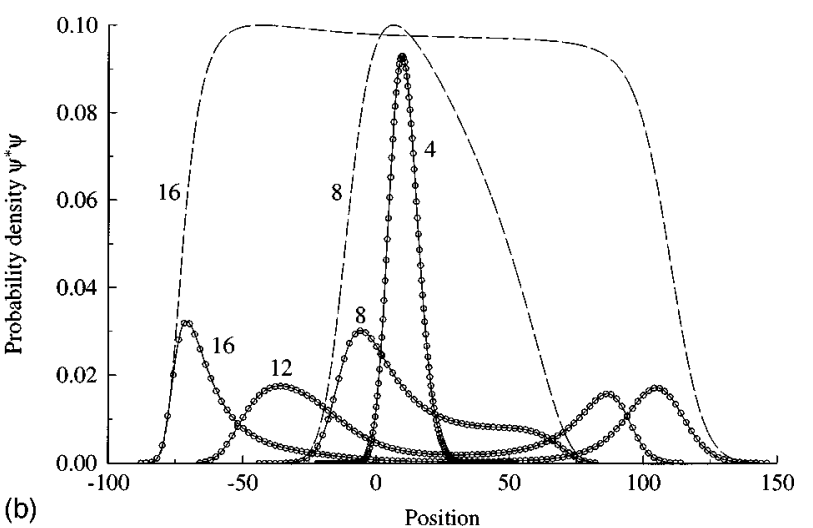

FIG. 6. The result of the quantum (solid) and semiclassical CTM (circles) propagations in the potential of Section IV C 2, at several times, indicated near the corresponding curve. (a) and (b) correspond respectively to the (t) and (r) cases, see Fig. 5(b). Notice the remarkable accuracy with which the CTM predicts the quantum wave packet for all times, in a situation far from a usual semiclassical regime. Dashed lines represent the results for the zeroth order only $(/ 10)$ of the CTM.
The following wave packets plotted show the beginning of the splitting. The CTM prediction remains very accurate even at this time. Note that the maxima observed do not correspond to the position of the classical particle associated with the wave packet at this time.

As time elapses, the splitting of the wave packets in two counter-propagative packets becomes more and more clear. The complex trajectory method achieves a very accurate prediction of the quantum wave packet, in a situation far from the ones that are usually relevant to semiclassical methods. At times $t=20$ and $t=18$ for the Figures 6(a) and 6(b), the comparison has been made between the real parts of the exact wave packet and the approximated one on one hand, and the imaginary parts on the other hand, the accuracy observed of the CTM is good as well. In Figs. 6(a) and 6(b), the wave packet calculated by means of the CTM restricted to the zeroth order (divided by a factor 10) is plotted at two times. This allows us to realize the importance of the first order correction relative to the zeroth one. In particular, it is easy to show that the maximum of the zeroth order probability density is located at the point reached by the real trajectory at the corresponding time. The real trajectory is thus always late relative to the position of the maximum of the transmitted wave packet. We can add that this example confirms very clearly the nonlinear nature of the CTM (i.e., it is not equivalent to the GWPPM) since a linear method of wave packet propagation would have predicted simply either the entire transmission or the entire reflection, in the $(\mathrm{t})$ and $(\mathrm{r})$ cases, respectively.

\section{CONCLUSION}

We shall stress again the main feature of the principle of the complex trajectory method as a semiclassical formalism, aimed at wave packet propagation. The amplitude of the wave packet is not treated as a corrective or first order term as it is done usually; instead it is included at the very beginning of the semiclassical expansion or at the zeroth order term. As a consequence, both the wavelength and the width of the wave packet tend to zero at the semiclassical limit, unlike in the WKB method. 
The examples reviewed, particularly the numerical ones, highlight the remarkable properties of the complex trajectory method. Not only is the accuracy of the WKB wave packet propagation method greatly improved but another unexpected and appealing property emerges: the disappearance of the singularities (caustics) that the real (WKB and VanVleck) semiclassical methods bring in, at least in the case of the potentials considered here. As stressed along these lines, the CTM is not a linear (GWPPM) theory of wave propagation. Thus, the complex trajectory method is a semiclassical theory that appears to be as accurate as the most accurate semiclassical theory known (the Van-Vleck formula) while being nearly as computationally simple as the most simple known one, the WKB.

Many approaches to semiclassical approximations including complex aspects rely on the analytical extension of the usual (real) semiclassical theory, as insisted before, the CTM is not of this type. W. H. Miller made classical trajectory calculations in the classically forbidden region of configuration space by means of complex numbers, in order to compute tunneling effect contribution to collision cross section. ${ }^{25}$ More recently, the introduction of trace formulas has allowed one to relate at the semiclassical limit the energy spectrum of a Hamiltonian to closed orbits of the phase space, in the integrable ${ }^{26}$ as in the chaotic case. ${ }^{13}$ The inclusion of complex classical trajectories leads to a better convergence of the trace formulae, in the regular $\operatorname{case}^{27}$ and in the irregular case. ${ }^{28}$

Consideration of the complex phase space to propagate Gaussian wave packets at the semiclassical limit has already been used by Heller and coworkers. ${ }^{29,30}$ Let us note at first that their method, denoted as GGWPPM, relies on a totally different principle to ours. It consists of extending the usual GWPPM to the complex phase space by noticing that the initial Gaussian wave function is not changed if its average position and momentum are transformed to complex values. A situation where the methods can be compared is the case of the bounce of a wave packet against a wall, when the packet is near the turning point. It shows oscillations in its probability density, due to interference of the two counterpropagative waves, and exponential decrease in the classically forbidden region, see Fig. 3 in this paper and Fig. 4 in Ref. 29. Huber and Heller consider double contributions, or double paths, to compute the propagated wave packet, hence, they are able to predict part of the oscillations of the wave packet. In this application of the CTM, we use the rootsearching procedure explained in Section IV A, excluding de facto such double contributions. The wave packet around the turning point is therefore not accurately predicted. Nevertheless, it appears that in the GGWPPM, all contributions to the wave packet diverge in the classically forbidden region ${ }^{31}$ whereas the CTM predicts its nearly zero value.

Whereas the GGWPPM relies on a complex GWPPM and a root-searching procedure, in our method, the property of analyticity of the computed wave packet can be used to extrapolate on the real line the wave function at the final time, avoiding the root-searching procedure, cf. Section II B. Moreover, when computing an overlap with another state or a matrix element in order to compute observable quantities, one can deform the original path of integration made up of the real line, to a complex line which interpolates the points reached by the trajectories, and extend easily the other analytical function involved in the integral to the same complex line. This allows us to make direct use of the computed values at complex points and even to avoid the extrapolation procedure itself in the computation of the integral. To end, let us add that two-dimensional wave packet propagations by means of the complex trajectory method have already been carried out. They are currently being studied and will be compared with numerical quantum calculations. The CTM can be generalized to any single linear partial differential equation, particularly time-dependent Hamiltonians can be considered. Note that the CTM can be used as easily within a discrete energy spectrum as within a continuum.

${ }^{1}$ F. Le Quéré and C. Leforestier, J. Chem. Phys. 94, 1118 (1991).

${ }^{2}$ D. H. Zhang and J. Z. H. Zhang, J. Chem. Phys. 101, 3671 (1994).

${ }^{3}$ D. H. Zhang and J. Z. H. Zhang, J. Chem. Phys. 101, 1146 (1994).

${ }^{4}$ D. Neuhauser, J. Chem. Phys. 10, 9272 (1994).

${ }^{5}$ H. Wadi and L. Wiesenfeld, Phys. Rev. E 55, 271 (1997).

${ }^{6}$ J. Palma and J. Echave, J. Chem. Phys. 104, 1146 (1996).

${ }^{7}$ L. L. Halcomb and D. J. Diestler, J. Chem. Phys. 84, 3130 (1986).

${ }^{8}$ N. Balakrishnan and G. D. Billing, J. Chem. Phys. 101, 2785 (1994).

${ }^{9}$ M. V. Berry and K. E. Mount, Rep. Prog. Phys. 35, 315 (1972).

${ }^{10}$ L. Landau and E. Lifshitz, Mécanique Quantique (Mir, Moscow, 1967).

${ }^{11}$ P. A. M. Dirac, The Principle of Quantum Mechanics, 4th ed. (Oxford University Press, Oxford, 1958).

${ }^{12}$ V. P. Maslov and M. V. Fedoriuk, Semi-classical Approximation in Quantum Mechanics (Reidel, Dordrecht, 1981); J. J. Duistermaat, Fourier Integral Operators (Birkhäuser, Boston, 1996).

${ }^{13}$ M. C. Gutzwiller, Chaos in Classical and Quantum Mechanics (SpringerVerlag, Berlin, 1990).

${ }^{14}$ E. J. Heller, J. Chem. Phys. 62, 1544 (1975).

${ }^{15}$ E. J. Heller, in Chaos and Quantum Physics, Les Houches, Session LII (North-Holland, Amsterdam, 1991).

${ }^{16}$ E. J. Heller, J. Chem. Phys. 94, 2723 (1991).

${ }^{17}$ I. M. Suarez Barnes, M. Nauenberg, M. Nockleby, and S. Tomsovic, Phys. Rev. Lett. 71, 1961 (1993); S. Tomsovic and E. J. Heller, ibid. 67, 664 (1991); Phys. Rev. E 47, 282 (1993).

${ }^{18}$ V. I. Arnold, Geometrical Method in the Theory of Ordinary Differential Equation, 2nd ed. (Springer-Verlag, Berlin, 1983).

${ }^{19}$ The condition is that $s$ and $t$ are one to one for all characteristics.

${ }^{20}$ G. Vattay, Prog. Theor. Phys. Suppl. 116, 251 (1994).

${ }^{21}$ A. M. Ozorio de Almeida, Hamiltonian Systems, Chaos and Quantization (Cambridge University Press, Cambridge, 1988).

${ }^{22}$ C. Cohen-Tannoudji, B. Diu, and F. Laloë, Mécanique Quantique (Hermann, Paris, 1973).

${ }^{23}$ M. Boiron, thesis, Grenoble, 1995. The result was obtained through space Fourier transforming the Schrödinger equation, solving the resulting first order equation by the method of characteristics and finally back Fourier transforming the previous solution. Note that a symbolic calculator can also be used.

${ }^{24}$ W. H. Press, B. P. Flannery, S. A. Teukolsky, and W. T. Vetterling, Numerical Recipes (Cambridge University Press, Cambridge, 1986).

${ }^{25}$ W. H. Miller and T. F. Georges, J. Chem. Phys. 56, 5668 (1972).

${ }^{26}$ M. V. Berry and M. Tabor, Proc. R. Soc. London A 349, 101 (1976).

${ }^{27}$ M. Joyeux, Chem. Phys. Lett. 247, 454 (1995).

${ }^{28}$ P. Leboeuf and A. Mouchet, Phys. Rev. Lett. 73, 1360 (1994).

${ }^{29}$ D. Huber and E. Heller, J. Chem. Phys. 87, 5302 (1987).

${ }^{30}$ D. Huber, E. Heller, and R. G. LittleJohn, J. Chem. Phys. 89, 2003 (1988).

${ }^{31}$ Each branch considered leads to a divergence, not only one while the other leads to a vanishing contribution. 